\title{
THE DETERMINATION OF THE THERMAL RELIABILITY CRITERION FOR BUILDING ENVELOPE STRUCTURES
}

\author{
Gennadiy FARENYUK
}

\begin{abstract}
The paper presents the basic methodical principles for the time analysis of the variations of envelope structures' thermal insulation properties and for the substantiation of the thermal reliability criterion, which should allow the analysis of the actual parameters of heat losses during the operation of buildings. In the paper, the state of the envelope structures thermal failure, the concept of building thermal envelope thermal reliability and the principles of its rating are defined. The physical meaning and basic criterion of the envelope structure thermal reliability are formulated. The application of the thermal reliability criterion allows determining the probable variations in the thermal insulation properties during the building operation and, accordingly, the changes of the building energy performance over time.
\end{abstract}

Keywords: building envelope structure; criterion; thermal reliability

\section{INTRODUCTION}

A modern building is a system composed of a number of subsystems that can be considered as independent systems. The system of enclosing structures, which is a thermal envelope of a building, is a determining factor in the context of defining the energy performance indicators for a building. It is necessary to develop the methodological guidelines for this system to determine reliability. The structural design of the elements of the building thermal envelope determines the structural response to external influences and the nature of the formation of the relevant operating indicators for a building.

The possible decrease of the thermal engineering indicators of the envelope structures depends on the features of the heat and mass transfer processes defined by the thermophysical properties of the materials and the structural design of the envelope, as well as on the critical states of moisture within the structure thickness causing the structural variations in materials and construction elements and, consequently, the energy state deterioration in the entire system.

The consideration of a building as an object of human activity allows singling out its two main functions, namely, the spatial and thermal ones. The spatial function of a residential building envisages the creation of a certain space where a person can form his/her private living environment fully according to his/her own free choice with a relatively minimal influence of the society. For production and civic buildings, the spatial function is to allocate a special volume for performing definite technological operations. The human body can function normally in a narrow temperature range, therefore, it is necessary to protect the human beings from adverse climatic influences and create a specific thermal and gas environment, which is the second functional task of the house. This function implementation is a rather complicated engineering task. For its solution, a building must be equipped with a variety of communications and systems, the stable operation of which directly ensures the reliable and no- failure operation of the building. In this context, the state of the building, when the performance of at least one of the above-mentioned functions is not ensured, can be considered as the failed state.

The building must meet three main criteria as follows: (1) the overall reliability, which defines the protection degree for a human being as an individual and as a social group; (2) the comfort, which sets the parameters of the thermal, acoustic, light, gas environment; (3) the cost effectiveness, which determines the costs for ensuring the first two criteria. Additionally, there are certain architectural requirements conditioned by national traditions, time trends, manufacturing capabilities, etc. All these parameters of the building are majorly determined by its thermal envelope, which is composed of a system of enclosing structures, the structural solutions of which define the building performance.

The paper's goal is to determine the basic methodological principles for the analysis of the time variations of the thermal insulation properties of the envelope structures and to justify the thermal reliability criterion in order to analyze the actual parameters of heat losses during the building's operation.

As a science, the theory of reliability arose in the 1960s1980s and was developed in the works of many scholars, including the Ukrainian specialists [1-7]. The provisions of theoretical studies, set forth in the referenced scientific works, were taken as a basis for the development of the rating method for the reliability of the buildings' thermal envelopes [8].

For the first time, the concept of the thermal reliability of envelope structures and the failures of building thermal insulation were considered in [9]. The failure as a reliability characteristic is an event that occurs when an object loses its ability to perform the assigned function in ensuring its serviceable condition. For a building, an event of failure is considered as a state when the fulfillment of prescribed (normalized or comfortable) heat and moist conditions in the building premises is not ensured. 


\section{THE VARIATIONS OF ENVELOPE STRUCTURES' THERMAL INSULATION PROPERTIES}

The method for rating the thermal reliability of envelope structures is based on the principle of the design boundary states' determination, when the requirement of failure-free operation, i.e., of non-excess beyond the boundary state, is established by the inequality of the following type:

$$
\Omega\left(w, T, R, R_{g}, z, \tau, \varepsilon, I, J, W\right)=\Omega\left(\Theta_{j}\right) \leq \frac{\Gamma_{\aleph}}{\wp_{\aleph}}
$$

where: $\Omega\left(\aleph_{j=1, \ldots, J}\right)$ is the parameter's function of the considered system (an envelope structure, a thermal envelope, etc.); $\left(\Theta_{j}\right)$ are the design values of the system's actions and characteristics; $\Gamma_{\aleph}$ - function limit by the operating parameter $\Theta_{j}$; and $\wp_{\aleph}$ - coefficient of reliability taking into account the sensitivity of a structure for its survivability under the variations of the corresponding parameter $\Theta_{j}$.

In the general theory of reliability, the construction failure [1] is seen as the crossing of the permissible region random boundary by a random path that reflects the construction behavior. The envelope structure behavior is characterized by a variation of its thermal insulation properties under the influence of climatic external factors and internal heat and moisture environment. Additionally, the installation factors also determine the random boundaries of the probable thermal state of the envelope structure.

The external influences affecting the thermal envelope and determining the limits of the boundary states of the enclosing structures include the following factors:

- the variations of the external and internal air temperature and humidity;

- atmospheric influences including solar radiation, rain, snow, wind; and

- the nature of temperature variations during the cold season.

The structural solutions of the enclosing structure and the thermal envelope of the building as a whole determine the structure response to the external influences and the nature of the corresponding operational indicators formation for the studied object. The probable reduction of the thermal engineering parameters of the enclosing structures depends on the specific features of the heat and mass transfer processes, which are defined by the thermophysical properties of the materials, the structural solution of the envelope, as well as the emergence of moisture critical states in the thickness of the structure, which lead to structural changes in materials and construction elements and, consequently, to the deterioration of the energy state of the general system. This causes the need for a thorough analysis of all components that affect the indicators of thermal reliability of structures and, accordingly, the energy performance of the building.
Failure is a condition which happens when object performance is interrupted. For enclosing structures, the state of thermal failure should be detected in the presence of at least one of the following parameters:

- a drop of the local values of the inner surface temperatures up to the temperature of air vapor condensation under the design thermal conditions (Fig. $1)$;

- accumulation of moisture in the thickness of the structure during the annual operating cycle (Fig. 2);

- the occurrence of layers of mold or fungal formations on the inner surface of the structure or under its decorative layers (Fig. 3-5).
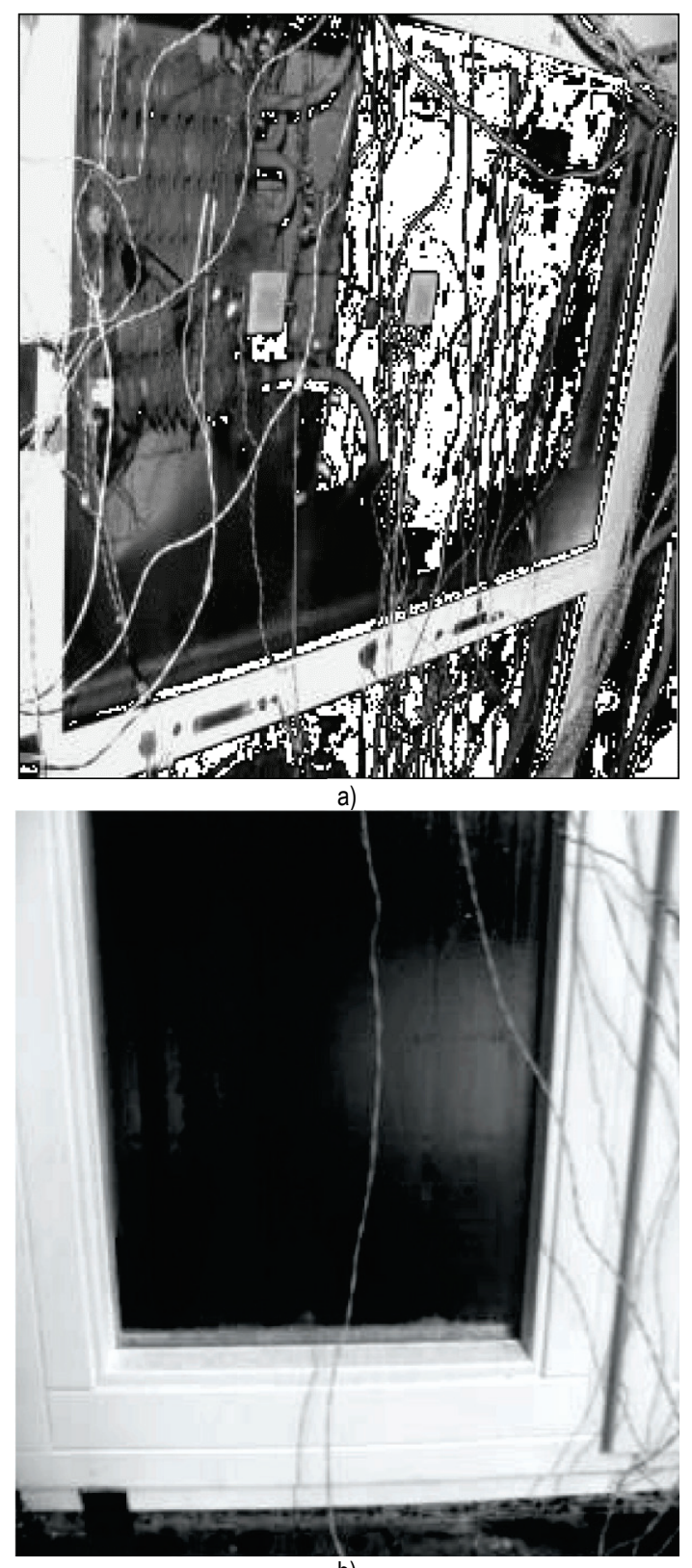

Figure 1 The condensate (a) and hoarfrost (b) formation on the surfaces of transparent structures during tests under the design temperature conditions in the climatic chamber. 


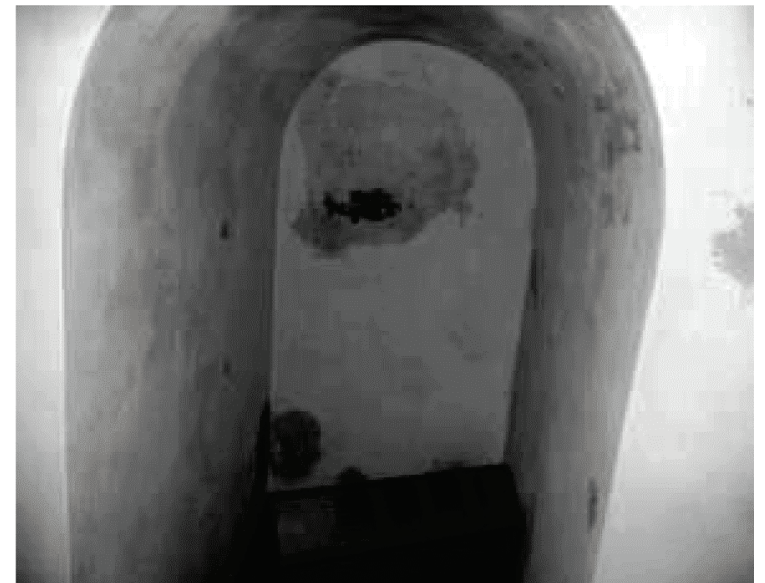

Figure 2 Accumulation of moisture in the external wall of the temple building during the annual operating cycle.

In its physical essence, the thermal failure of structures, thermal envelopes or buildings as a whole relates to partial failures, that is, to the loss of the structures' ability to perform the part of their functions. In the case of a thermal envelope thermal failure, a building does not lose its characteristics of the overall reliability, including the environmental protection of the people, etc. However, in such circumstances, the above listed criteria of the building's operational suitability are not met.

The thermal failure concept is directly related to the notion of the structure boundary state and in our case, it should be considered as the state of the building thermal envelope, in which further building operation is associated with the violations of normal human life conditions, increased economic costs and the structures' durability decrease.

The damage of some elements in civil structures affects the operational reliability of other elements of the unit. This general principle applies to the thermodynamic processes in multi-layered enclosing structures, in which, for instance, the failure in a form of a breach of vapor sealing properties of the corresponding structural layer results in the decrease of the insulating properties of the warmth-keeping layer. This makes it necessary to assess structure reliability, when the modern enclosing structure is considered as a system, the design and manufacture of which was carried out according to functional requirements [10], taking into account the mutual influences of its elements.

In the process of considering the thermal envelope of a whole building as a system, this principle remains unchanged and envisages that the failure of an envelope element (for instance, a window structure) leads to thermal damages of the wall opaque structures and of the entire system.

Therefore, the reliability rating for an object consisting of a system of interacting elements, such as a multilayered thermally heterogeneous structure or a building thermal envelope, should obviously be based on the characteristics of individual elements and the use of the statistical simulation method $[2,6]$. In this case, the functioning of the system is presented as a sequence of random events associated with the individual elements of the system. The event points out any deterioration of the element operation quality. In the process of simulation, the interrelation of elements in the system is established by changing the characteristics of individual elements after the event for any of them. The simulation is carried out for a specific period, for instance, for the estimated warranty period, and so on.

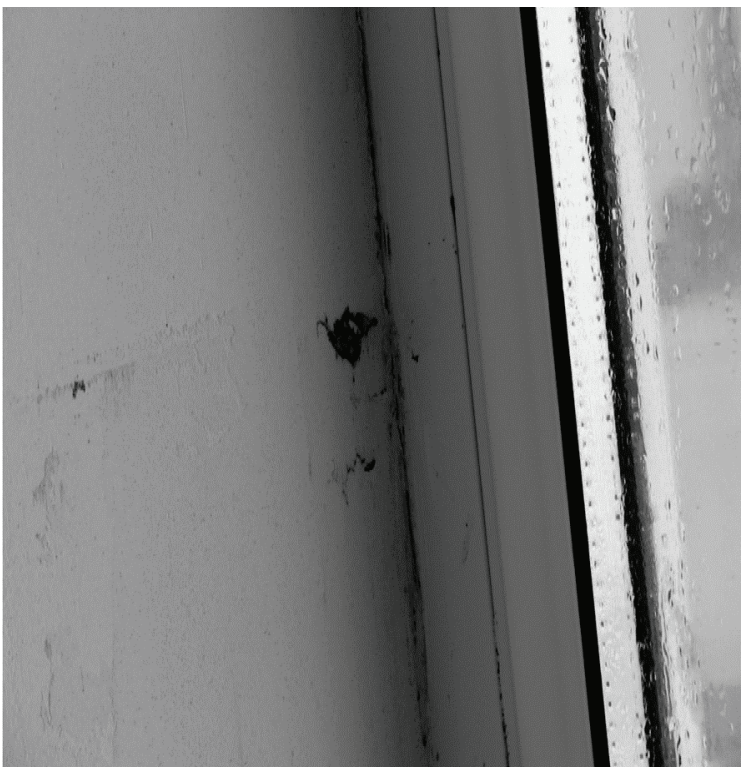

a)

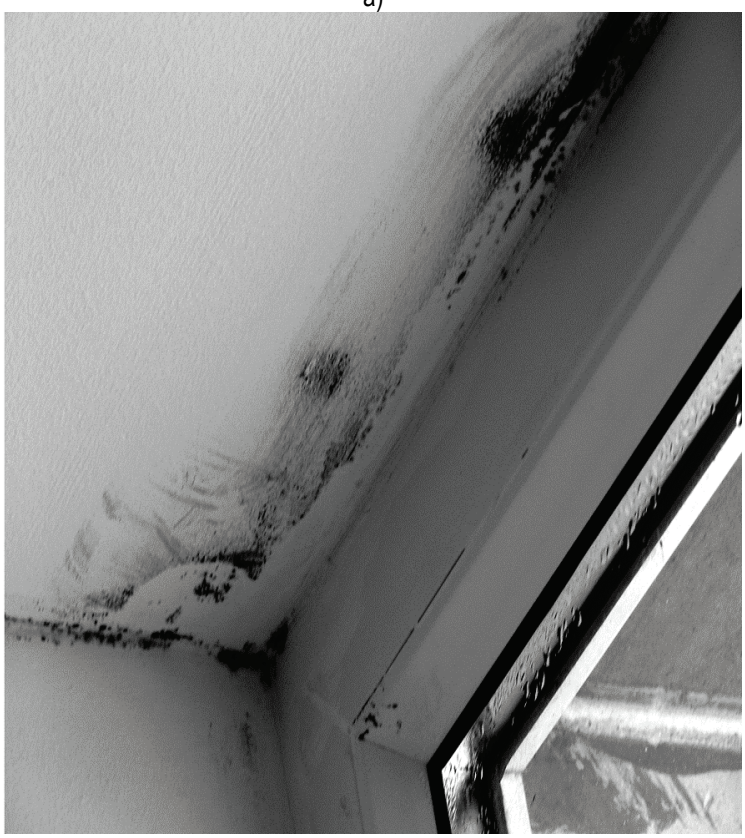

b)

Figure 3 The states of thermal failures on the surfaces of the walls (a) and ceiling (b) of the living-room during the first cold period after the replacement of old windows with modern PVC windows with a one-chamber glass unit (4M-16-4M).

Every single simulation cycle can be reiterated, and the statistics of the system in various states can be collected with the various quantities of events for system elements. Based on such statistics, the reliability of the system is rated.

The simplest scheme of element connection is a multilayered construction consisting of elements connected in a series according to the heat flow resistance. In 
compliance with the technical theory of reliability, the reliability-rating is carried out by the characteristic of the probability of a system failure-free operation with independent elements by means of the following equation:

$$
P_{s}=\prod_{i=1}^{n} P_{i}
$$

where $P_{i}$ is the probability of an $i^{\text {th }}$ element of a failure-free operation.

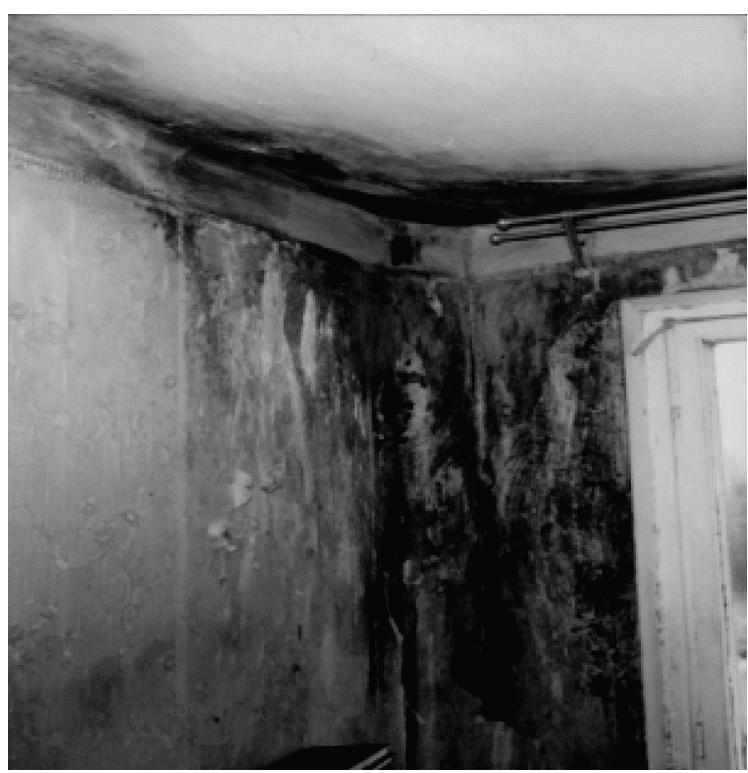

a)

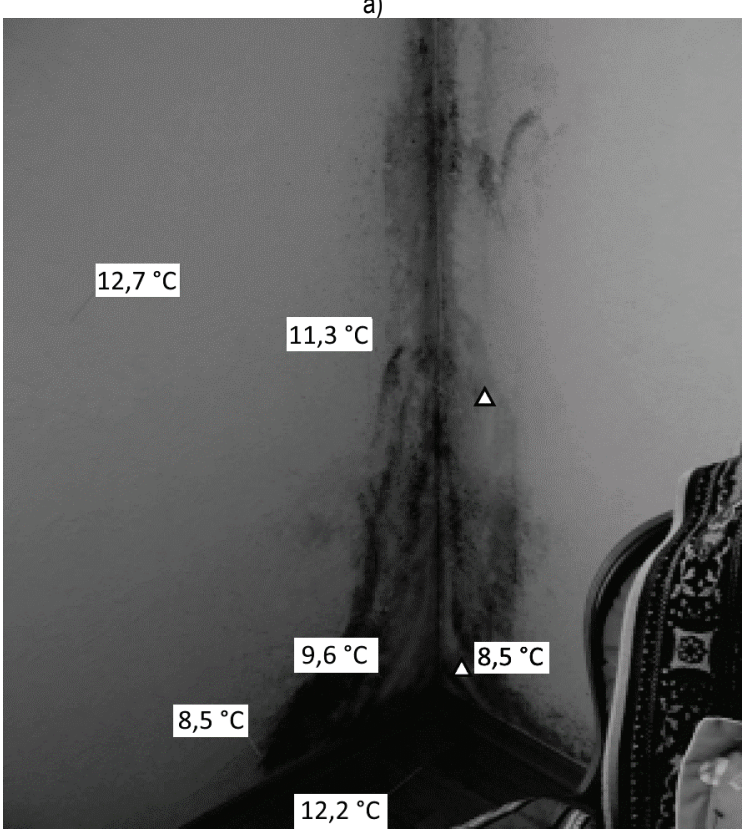

b)

Figure 4 The states of thermal failures at the corner areas of apartments

In the processes of heat transfer through the structure, the sequential scheme envisages a thermally homogeneous envelope structure. Real structures, especially the modern ones, are thermally heterogeneous, and the heat transfer is carried out according to a scheme that can be considered as two (or more) groups of series-connected elements on the assumption that the groups are connected in a parallel (Fig. 6 ), and the estimation of the failure-free operation probability of the system is carried out according to the Eq. (3).

$P_{S}=1-\prod_{i=1}^{n}\left(1-P_{i}\right)$

The operational reliability of individual elements is characterized by the probability $P_{i}$ of a failure-free operation of the corresponding element during the given period in the conditions, when other elements operate normally. An expert assessment is used for this characteristic. A set of respective probabilities for all elements constitutes the vector of reliability coefficients.
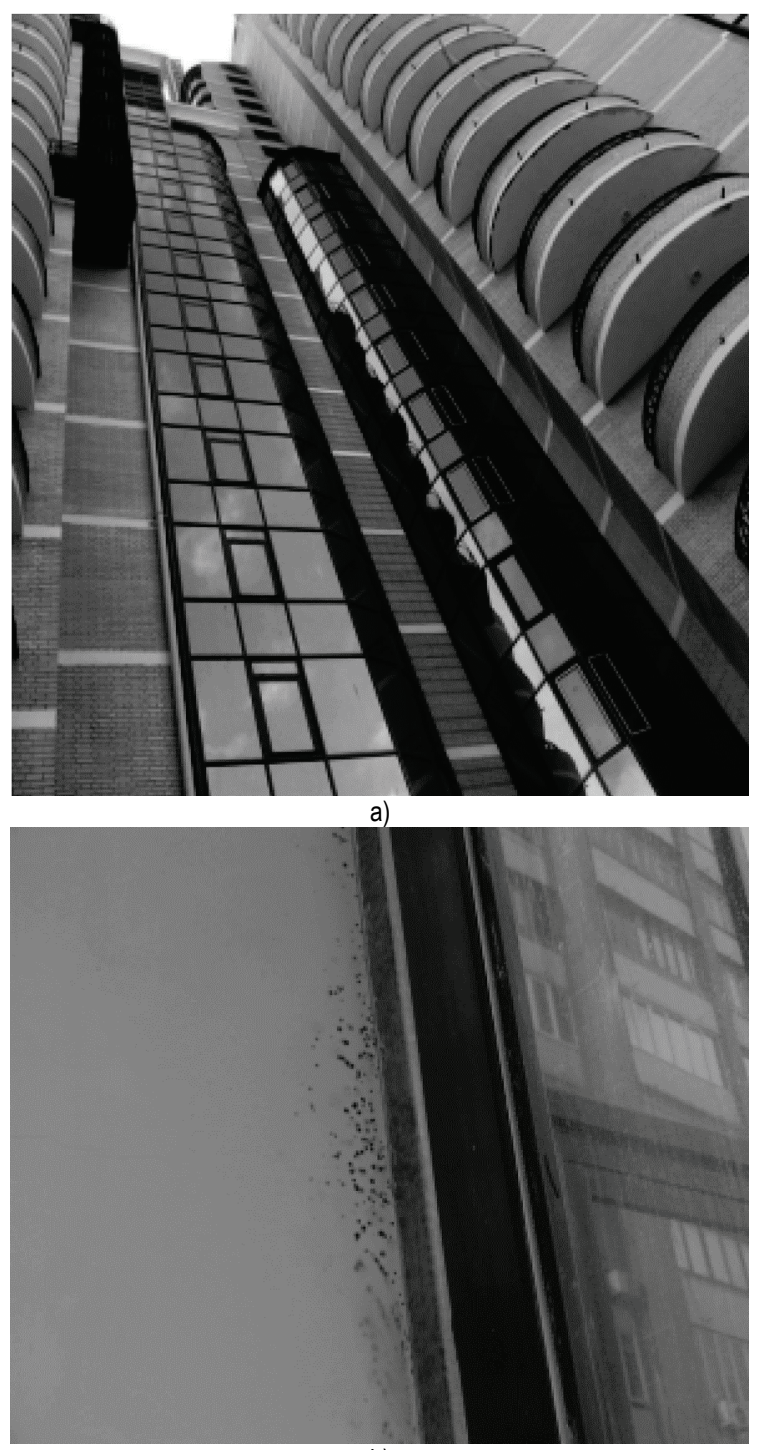

b)

Figure 5 Appearance of the façade element in a building (a) having the recorded state of the external walls' thermal failures after three months of operation (b). 


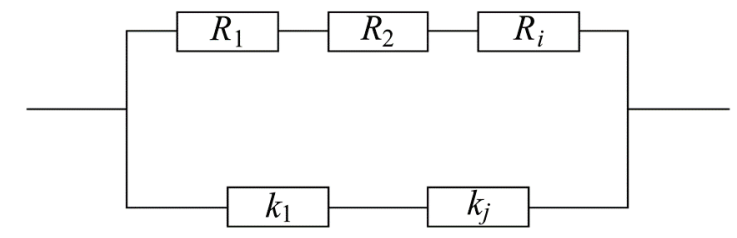

Figure 6 Conditional representation of the thermal work of thermal envelope structures $\left(R_{1}, R_{2}\right.$ and $R_{i}$ are the thermal resistances of the $1^{\text {st }}, 2^{\text {nd }}$ and $i^{\text {th }}$ layers of the structure; $k_{1}$ and $k_{j}$ are the thermal transfer linear coefficients of the $1^{\text {st }}$ and $j^{\text {th }}$ thermally conductive connections).

\section{CRITERION OF THE THERMAL RELIABILITY OF THE ENVELOPE STRUCTURES}

The assessment of the thermal insulation reliability has its own features. In radio engineering systems, the failure of one element results in system failure. The study of the reliability of load-bearing civil structures shows that the failure of one element can lead to the failure of the system. In this case, the element fail occurs once, and this failure is decisive for the system's further operability. In envelope structures, the thermal failures of elements may occur many times during the given time. The failures of the entire system can also be multiple. If this occurs, the construction does not lose its heat-insulating properties completely. Therefore, for assessing the thermal reliability of enclosing structures, it is insufficient to determine the failure-free operation probability and other frequency characteristics, but indicators that characterize the possibility of the changes of the building thermal envelope operational properties should be introduced.

The possible variations of the main characteristics of the thermal insulation, i.e., of the reduced total thermal resistance, can be taken as a criterion for the reliability of the system (building envelope, enclosing structure, etc.):

$R_{\mathrm{r}}=1-\frac{R_{\Sigma \mathrm{pr}}(0)-R_{\Sigma \mathrm{pr}}(N)}{R_{\Sigma \mathrm{pr}}(0)}$

where $R_{\Sigma \mathrm{pr}}$ is the reduced total thermal resistance of the enclosing structure.

The physical essence of this criterion is the determination of the envelope structure operational efficiency during its predetermined period of operation under the possible changes of the state of the structural elements because of the influence of various (climatic, assembling, operational, etc.) factors. The criterion can vary from one to near-zero values. In the first boundary state, the enclosing structure fully retains its thermal insulation properties during its predetermined lifetime. In the second boundary state, it completely loses its thermal insulation properties and is no longer an envelope structure, although it physically exists and is an obstacle to the external environment. The criterion of the reduced total thermal resistance characterizes the reliability of the building thermal insulation, because its value is determined by taking into account the effect of the possible failures of each element of the structure on the occurrence of failures in other elements and the corresponding change of the insulation properties of the structure (the system under consideration) after the cycle of probable climatic influences.

\section{CONCLUSIONS}

The developed method application for the statistical simulation of the processes of the heat failure of building thermal envelopes provides for the possibility for a quantitative comparative assessment of the design principles used in the modern construction sector. The proposed criterion of thermal reliability allows to assess the probable change of the thermal insulation indicators of the envelope structures' system by taking into account the effect of the possible failures of each system element on the occurrence of failures in other elements, and the corresponding change of the thermal insulation properties of the system after the cycles of probable climatic actions.

\section{REFERENCES}

[1] Bolotin, V. V. (1982). Methods of probability theory and reliability theory in buildings design, Stroiizdat, Moscow, 351 $\mathrm{p}$.

[2] Ventsel, E. S. (1988). Theory of probability and its engineering applications, Moscow, $479 \mathrm{p}$.

[3] Levin, B. R. (1978). Theory of radio engineering systems (mathematical basis), Sovietskoie radio, Moscow, $263 \mathrm{p}$.

[4] Lloid, D. (1964). Reliability, Sovietskoie radio, Moscow, $685 \mathrm{p}$.

[5] Perelmuter, A. V. (2000). Selected problems of civil structures reliability and safety, $2^{\text {nd }}$ revised ed., Stal, Kyiv, 216 p.

[6] Pichugin, S. F. (2009). Civil structures reliability, Poltava, ASMI, $452 \mathrm{p}$.

[7] Kaufman, Ar. (1969). La confiance technique. Theorie mathematique de la frabelitie, Dunod, Paris, $79 \mathrm{p}$.

[8] Farenyuk, G. G. (2009). Basics of assuring the buildings energy performance and envelopes thermal reliability, GamaPrint, Kyiv, 216 p.

[9] Farenyuk, G. G. (1983). Experimental investigation of rizopen phenolic plastic foam durability. Investigations of buildings thermal protection, NIISF, Moscow, 93-99.

[10] Farenyuk G. G. (2007). Functional design of modern envelope structures. Construction materials, products and sanitary engineering, 7, 109-113.

\author{
Authors' contacts: \\ Gennadiy FARENYUK, Doctor of Engineering Sciences \\ Odessa State Academy of Civil Engineering and Architecture, \\ Ukraine, 65029, Odessa, 4 Didrihsona str. \\ State enterprise "State Scientific Research Institute of Building Constructions", \\ Ukraine, 03037, Kyiv city, 5/2 Preobrazhenska str. \\ Phone: 0662803754 \\ E-mail: farenyuk@ndibk.gov.ua
}

